\title{
Torsional Vibration Analysis of Carbon Nanotubes Using Maxwell and Kelvin-Voigt Type Viscoelastic Material Models
}

\author{
Mustafa Arda \\ ${ }^{1}$ Trakya University, Department of Mechanical Engineering, 22130, Edirne, Turkey
}

\begin{abstract}
Torsional dynamic analysis of viscoelastic Carbon Nanotubes (CNT) has been carried out in the present work. Maxwell and Kelvin-Voigt type viscoelasticity are considered in the modeling of viscoelastic material. Nonlocal Elasticity Theory is used in the formulation of governing equation of motion and boundary conditions. Viscoelasticity and nonlocal effects of structure on the free torsional vibration of CNTs have been investigated. Clamped-clamped and clamped-free boundary conditions are considered. Present study results could be useful in design of nano-medicine delivery applications.
\end{abstract}

Key Words: Viscoelastic Material, Maxwell, Kelvin-Voigt, Carbon Nanotubes, Torsional Vibration, Nonlocal Elasticity.

\section{INTRODUCTION}

Carbon Nanotubes (CNTs) have a potential about being a new functional material for engineering problems which could not be solved with traditional materials. With their superior physical properties, CNTs are an important candidate for engineering application in medical industry. Delivering a drug to tissue or cell could be achieved with torsional movement of CNTs. An external effect like magnetic field can rotate the nanotube and release the medicine. Torsional modeling of CNTs in such applications has great importance.

CNTs can be modelled with continuum mechanic theories like nonlocal elasticity, strain gradient, modified couple stress, doublet mechanics or peridynamics theories. As a second approach, discrete models like molecular dynamics and lattice dynamics can be used. But, the classical continuum mechanics could not consider the size effect. Especially in nano dimension, size dependency gains much importance because of atomic interactions. Differently from classical continuum mechanics theory, Nonlocal Elasticity [1,2] considers size dependencies.

Peddieson et al. [3] firstly proposed the nonlocal Euler-Bernoulli beam model for CNTs. Wave propagation analysis in CNTs were carried out with using nonlocal models by [4-6]. Nonlocal constitutive relations of Eringen was reformulated by several researchers and various beam theories were obtained in [7-9].
CNTs generally have modeled as an elastic structure but in reality viscous characteristics of CNTs have been seen. For more realistic approach, CNTs must be assumed as a viscoelastic structure. In literature search, studies about viscoelastic CNT modeling can be found but in majority of papers an elastic structure assumption have been considered. Ansari and Ajori [10] carried out the MD simulation of double-walled Carbon and Boron-Nitride hybrid nanotubes. Static and dynamic analysis of nanorods [11], microbeams [12] and functionally graded Rayleigh beams [13] were investigated by researchers. Firstly Chang and Lee [14] modeled the nonlocal model for viscoelastic CNTs with thermal and elastic foundation effects. Lei et al. [15] analyzed the dynamic behavior of viscoelastic Kelvin-Voigt CNTs with nonlocal Timoshenko beam model. Avcar [16] studied the free vibration of axially loaded beams resting on Pasternak Foundation. Karlicic et al. [17] investigated axial magnetic field effect on dynamics of the nanocomposites which were consist of multiple viscoelastic nanotubes and polymer as a matrix material. Arani et al. [18] studied the dynamics of fluid conveying viscoelastic CNTs with two dimensional magnetic field effect. Farokhi and Gayesh [19] investigated the shear deformable viscoelastic microbeams with modified couple stress theory. Cajic et al. [20] proposed a fractional viscoelastic model for CNT with attached particle problem. Ansari et al. [21] used the fractional order viscoelastic model with nonlocal Timoshenko beam model for free vibration of nanotubes. Zhang et al. [22] made the free vibration analysis of 
viscoelastic CNT embedded with viscoelastic media. Zhen and Zou [23] investigated the thermal and magnetic field effect on wave propagation in fluid conveying viscoelastic CNTs with using nonlocal strain gradient theory. Attia and Mahmoud [24] modeled the surface effect and nonlocality in viscoelastic nanobeams with modified couple stress and nonlocal elasticity theory. Cajic et al. [25] studied the free damped vibration of fractional order viscoelastic CNTs embedded in viscoelastic media with nonlocal elasticity model. Wang and Shen [26] used the nonlocal strain gradient theory in nonlinear vibration analysis of axially moving viscoelastic nanobeam. Naghinejad and Ovesy [27] used the finite element formulation and nonlocal integral elasticity in free vibration analysis of viscoelastic CNTs. Martin [28] proposed a nonlocal fractional Zener model for dynamic analysis of viscoelastic nanotubes. Pavlovic et al. [29] studied the stability of viscoelastic CNTs with dynamic axial loadings using higher order nonlocal strain gradient theory. Farajpour et al. [30] investigated the effects of viscoelasticity and geometrical imperfections on the nonlocal coupled linear and nonlinear mechanics of CNTs.

Torsional static and dynamic analysis of CNTs have been investigated in several papers [31,32]. But, in all these studies viscous characteristic of structure has not been considered. In this study, torsional viscoelastic CNT model is presented with using two different material assumption: Maxwell and Kelvin-Voigt. Nonlocal elasticity is used in obtaining the governing equation of motion and boundary conditions. Effect of viscoelasticity parameter and nonlocal parameter to the torsional dynamics of CNTs have been investigated. According to author's best literature knowledge, present topic has not been studied yet.

\section{ANALYSIS}

A viscoelastic $C N T$ of length $\mathrm{L}$ and diameter $\mathrm{d}$ is considered in clamped-clamped and clamped-free boundary conditions (Figure 1). Governing equation of motion for torsional behavior of hollow rod can be written as [33]torsional statics and dynamics of Carbon Nanotubes (CNTs:

$$
G I_{p} \frac{\partial^{2} \theta}{\partial x^{2}}=\rho I_{p} \frac{\partial^{2} \theta}{\partial t^{2}}
$$

where $G$ is the shear modulus, $\rho$ is the density, $I_{P}$ is the polar moment of inertia, $\theta$ is the angular displacement of CNT. The $I_{P}$ is defined as:

$$
I_{p}=\pi \frac{\left(R_{2}^{4}-R_{1}^{4}\right)}{2}
$$

where $R_{1}$ and $R_{2}$ are the inner and outer radius of CNT, res- pectively.

\subsection{Nonlocal Elasticity Theory}

The general differential form of the nonlocal constitute relation can be given as $[1,2]$ :

$$
\left(1-\nabla^{2}\right) \tau_{k l}=\frac{E_{v}}{(1+v)(1-2 v)} \varepsilon_{r r} \delta_{k l}+\frac{E}{(1+v)} \varepsilon_{k l}
$$

where $\tau_{k l}$ is the nonlocal stress tensor, $\varepsilon_{r r}$ is the sum of normal strains $\left(\varepsilon_{r r}=\varepsilon_{11}+\varepsilon_{22}+\varepsilon_{33}\right), \delta_{k l}$ is the kronecker delta, $\varepsilon_{k l}$ is the strain tensor, $v$ is the Poisson's ratio, $\mu=\left(e_{0} a\right)^{2}$ is called the nonlocal parameter, $a$ is an internal characteristic length and $e_{0}$ is a constant. $e_{0}$ is very important for the validity of nonlocal models. Eringen [1,2] determined this parameter with matching the dispersion curves based on the atomic models.

For the torsional deformation of uniform CNT, Eq. (3) can be written in one dimensional form:

$$
\left(1-\mu \frac{\partial^{2}}{x^{2}}\right) \tau=G \gamma
$$

where $\gamma$ is the shear strain, $\tau$ is the shear stress of CNT. The shear stress and torque resultants are expressed as:

$$
\begin{aligned}
& S=\int_{A} \tau d A \\
& T=\int_{A} \tau z d A
\end{aligned}
$$

By using the Eqs. (4-5), the constitute relation can be obtained as:

$$
\begin{aligned}
& S-\mu \frac{\partial^{2} s}{\partial x^{2}}=G A \gamma \\
& T-\mu \frac{\partial^{2} T}{\partial x^{2}}=G I_{P} \frac{\partial \theta}{x}
\end{aligned}
$$

If Eq. (6b) is inserted into Eq. (1) one obtains:

$$
G I_{P} \frac{\partial^{2} \theta}{\partial x^{2}}=\left(1-\mu \frac{\partial^{2}}{\partial x^{2}}\right) \rho I_{P} \frac{\partial^{2} \theta}{\partial t^{2}}
$$

Eq. (7) is the nonlocal governing equation of motion for the torsional deformation of elastic CNT. If the nonlocal parameter is chosen as zero $(\mu=0)$ in Eq. (7), the classical continuum mechanics equation is obtained. In the present study two different viscoelastic material type have been used in the analysis: Maxwell and Kelvin Voigt materials.

\subsection{Maxwell Type Viscoelastic Material}

Maxwell viscoelasticity model consist of serially connected purely viscous damper and elastic spring (Figure 2a). The stress-strain relation according to Maxwell viscoelastic material can be interpreted as [34]:

$$
\tau+\eta \frac{\partial \tau}{\partial t}=\eta G \frac{\partial \gamma}{\partial t}
$$

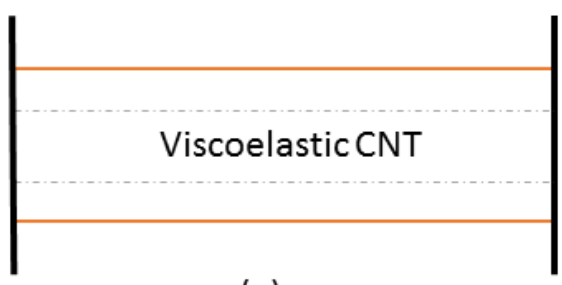

(a)

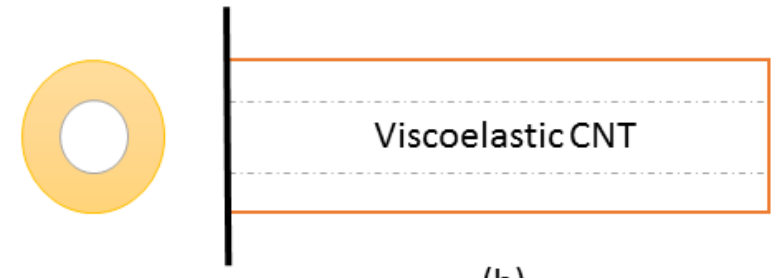

(b)

Figure 1. Viscoelastic CNTs: a)C-C and b)C-F 


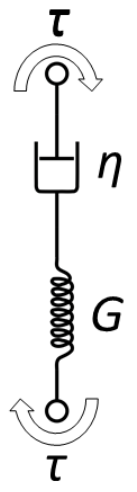

a)

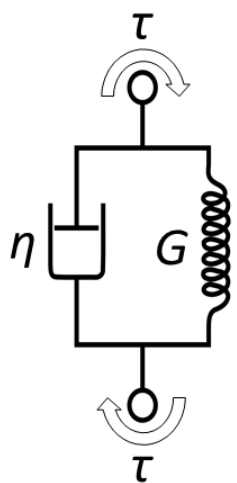

b)
Figure 2. Modeling of Viscoelastic Materials: a) Maxwell and b) Kelvin-Voigt

where $\eta$ is the dimensionless viscoelastic parameter. If Eq. (8) puts into nonlocal shear stress and torque relations, torsional governing equation of motion for the Maxwell viscoelastic CNT can be obtained as:

$$
\eta G I_{P} \frac{\partial^{3} \theta}{\partial x^{2} \partial t}=\left(1-\mu \frac{\partial^{2}}{\partial x^{2}}\right) \rho I_{P} \frac{\partial^{2} \theta}{\partial t^{2}}+\left(1-\mu \frac{\partial^{2}}{\partial x^{2}}\right) \eta \rho I_{P} \frac{\partial^{3} \theta}{\partial t^{3}}(9)
$$

Angular deformation of the Maxwell viscoelastic CNT can be assumed as below:

$$
\theta(x, t)=\varphi_{1}(x) e^{\lambda t}
$$

where $\phi_{1}(x)$ is the amplitude of torsional displacement and $\lambda$ is the characteristic parameter. If Eq. (9) is reorganized according to dimensionless parameter $\left(\bar{x}=\frac{x}{L}\right)$ assumption, governing equation of motion turns into the non-dimensional form as below:

$$
\frac{\partial^{2} \varphi_{1}}{\partial \bar{x}^{2}}\left[\eta \lambda+\lambda^{2} \frac{\mu}{L^{2}} \Omega+\eta \lambda^{3} \frac{\mu}{L^{2}} \Omega\right]-\varphi_{1}\left[\lambda^{2} \Omega+\eta \lambda^{3} \Omega\right]=0
$$

where $\Omega$ is the coefficient of characteristic parameter defined as:

$$
\Omega=\frac{\rho L^{2}}{G}
$$

If Eq. (11) is reorganized, one obtains:

$$
\frac{\partial^{2} \varphi_{1}}{\partial \bar{x}^{2}}-\beta_{1}^{2} \varphi_{1}=0
$$

where $\beta_{1}$ can be defined as:

$$
\beta_{1}=\sqrt{\frac{\lambda^{2} \Omega(1+\eta \lambda)}{\eta \lambda+\lambda^{2} \frac{\mu}{L^{2}} \Omega(1+\eta \lambda)}}
$$

Solution of the differential equation (13) can be expressed as:

$$
\varphi_{1}(\bar{x})=C_{1} e^{\beta_{1} \bar{x}}+C_{2} e^{-\beta_{1} \bar{x}}
$$

where $C_{1}$ and $C_{2}$ are the integration constants which can be defined using boundary conditions. In the present study, clamped-clamped (C-C) and clamped-free (C-F) boundary conditions are considered. If the elastic CNT boundary conditions in [33]torsional statics and dynamics of Carbon $\mathrm{Na}$ notubes (CNTs reformulated according to Maxwell viscoelastic material, boundary conditions for the present problem are interpreted as:

$$
\begin{aligned}
& \left.\begin{array}{l}
x=0 \rightarrow \theta(0)=0 \\
x=L \rightarrow \theta(L)=0
\end{array}\right\} C-C \\
& \left.\begin{array}{l}
x=0 \rightarrow \theta(0)=0 \\
\bar{x}=1 \rightarrow \eta G I_{P} \frac{\partial^{2} \theta}{\partial x \partial t}-\mu \rho I_{P} \frac{\partial^{3} \theta}{\partial x \partial t^{2}}-\mu \eta \rho I_{P} \frac{\partial^{4} \theta}{\partial x \partial t^{3}}=0
\end{array}\right\} C-F
\end{aligned}
$$

If the boundary conditions in Eqs. (15a) and (15b) are written in matrix form, homogenous system of linear equations could be solved for the characteristic parameter $(\lambda)$ which is the eigen-value of present problem. Its imaginary part can be defined as the non-dimensional damping (NDD) and real part can be defined as the non-dimensional frequency (NDF) of viscoelastic CNT, respectively. Integration constants $\left(C_{1}\right.$ and $\left.C_{2}\right)$ which are eigen-vector of present problem, can be determined if the linear systems of equations are solved for the corresponding characteristic parameter.

\subsection{Kelvin-Voigt Type Viscoelastic Material}

Kelvin-Voigt viscoelasticity consist of parallel connected purely viscous damper and elastic spring (Fig. (2b)). The stress-strain relation according to Kelvin-Voigt viscoelastic material can be interpreted as [34]:

$$
\tau=G\left(1+\eta \frac{\partial}{\partial t}\right) \gamma
$$

If Eq. (16) inserted into nonlocal shear stress and torque relations, torsional governing equation of motion for the Kelvin-Voigt viscoelastic CNT can be obtained as:

$$
G\left(1+\eta \frac{\partial}{\partial t}\right) I_{P} \frac{\partial^{2} \theta}{\partial x^{2}}=\left(1-\mu \frac{\partial^{2}}{\partial x^{2}}\right) \rho I_{P} \frac{\partial^{2} \theta}{\partial t^{2}}
$$

If Eq. (17) is reorganized using dimensionless parameter and angular deformation assumption in Eq. (10), governing equation of motion turns into the non-dimensional form as below:

$$
\frac{\partial^{2} \varphi_{2}}{\partial \bar{x}^{2}}\left[1+\eta \lambda+\lambda^{2} \frac{\mu}{L^{2}} \Omega\right]-\theta\left[\lambda^{2} \varphi_{2}\right]=0
$$

After reorganization of Eq. (18), one obtains:

$$
\frac{\partial^{2} \varphi_{2}}{\partial \bar{x}^{2}}-\beta_{2}^{2} \varphi_{2}=0
$$

where $\beta_{2}$ can be defined as:

$$
\beta_{2}=\sqrt{\frac{\lambda^{2} \Omega}{1+\eta \lambda+\lambda^{2} \frac{\mu}{L^{2}} \Omega}}
$$

Solution of the differential equation in Eq. (19) can be expressed as:

$$
\varphi_{2}(\bar{x})=C_{3} e^{\beta_{2} \tilde{x}}+C_{4} e^{-\beta_{2} \bar{x}}
$$

where $C_{3}$ and $C_{4}$ are the integration constants which should be defined for boundary conditions. Clamped-clamped (CC) and clamped-free (C-F) boundary conditions are reformulated according to Kelvin-Voigt viscoelastic material as below:

$$
\begin{aligned}
& \left.\begin{array}{l}
x=0 \rightarrow \theta(0)=0 \\
x=L \rightarrow \theta(L)=0
\end{array}\right\} C-C \\
& \left.\begin{array}{l}
x=0 \rightarrow \theta(0)=0 \\
\bar{x}=1 \rightarrow G I_{P} \frac{\partial \theta}{\partial x}+\eta G I_{P} \frac{\partial^{2} \theta}{\partial x \partial t}-\mu \rho I_{P} \frac{\partial^{3} \theta}{\partial x \partial t^{2}}=0
\end{array}\right\} C-F
\end{aligned}
$$

If the boundary conditions in Eq. (22a) and (22b) are written in matrix form, homogenous systems of linear equations 
could be solved for the characteristic parameter $(\lambda)$ which is the eigen-value of present problem. Integration constants which are eigen-vector of present problem, can be determined if the matrix form is solved for the obtained characteristic parameter. Damping ratio could be used as a characteristic value for viscoelastic structure and can be formulated using NDD and NDF values as below:

$$
\xi=\frac{|N D D|}{\sqrt{N D F^{2}+N D D^{2}}}
$$

\section{NUMERICAL RESULTS AND DISCUSSION}

In this section, torsional vibration analysis of viscoelastic CNTs carried out for various values of nonlocal parameter, viscoelasticity parameter and damping ratio. Validation of present torsional nonlocal elastic rod model has been investigated by present author in [33]torsional statics and dynamics of Carbon Nanotubes (CNTs with comparing the torsional one-dimensional Lattice Dynamics wave propagation results.

In Figs. (3) and (4), nonlocal effect on NDF and NDD of viscoelastic carbon nanotube in $\mathrm{C}-\mathrm{C}$ and $\mathrm{C}$-F boundary conditions can be seen, respectively. Maxwell material damping characteristics is not affected by nonlocality but stiffness of structure reduces and frequency decreases with nonlocal parameter. In Kelvin-Voigt material assumption, damping is decreasing with nonlocal effect and frequency firstly increases and then decreases with nonlocal parameter effect. Because of the fully clamped boundary condition on both ends, stiffness of nanotube slightly increases and then increasing nonlocal parameter shows softening effect on structure.
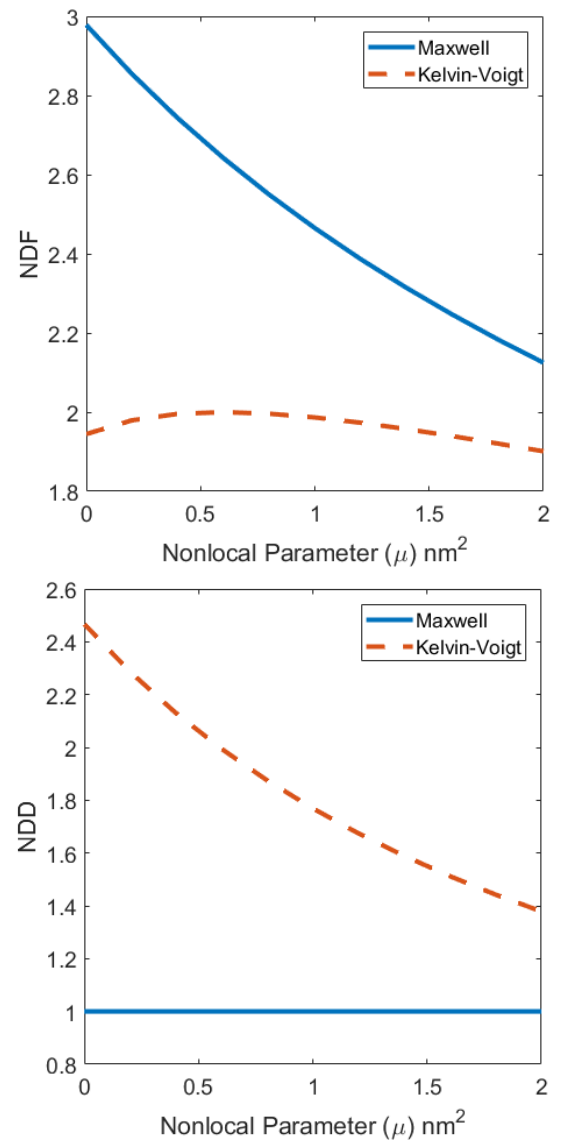

Figure 3. Nonlocal effect on NDF and NDD of C-C nanotube $(\eta=0.5)$
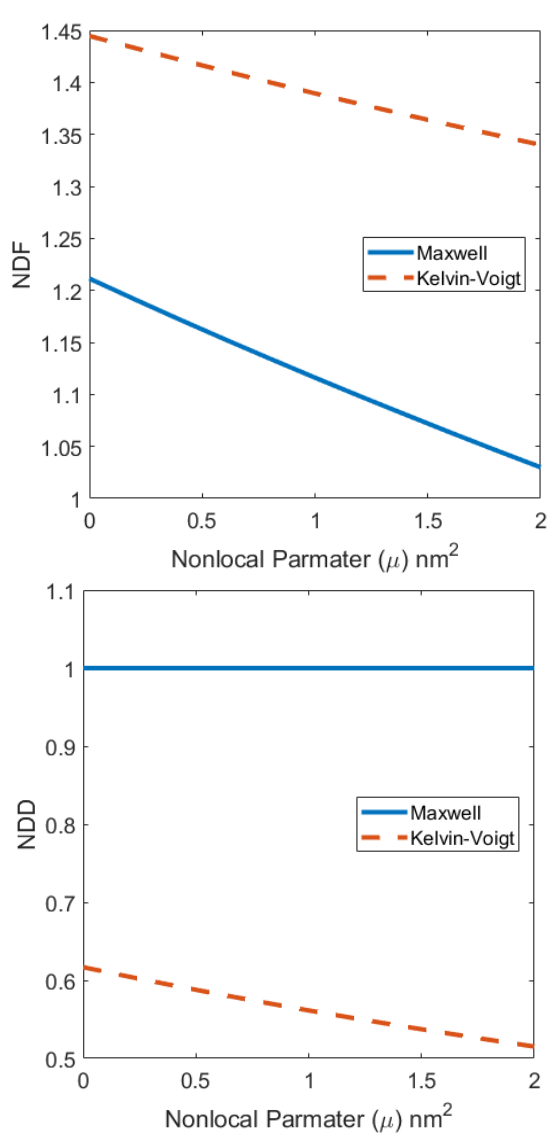

Figure 4. Nonlocal effect on NDF and NDD of C-F nanotube $(\eta=0.5)$
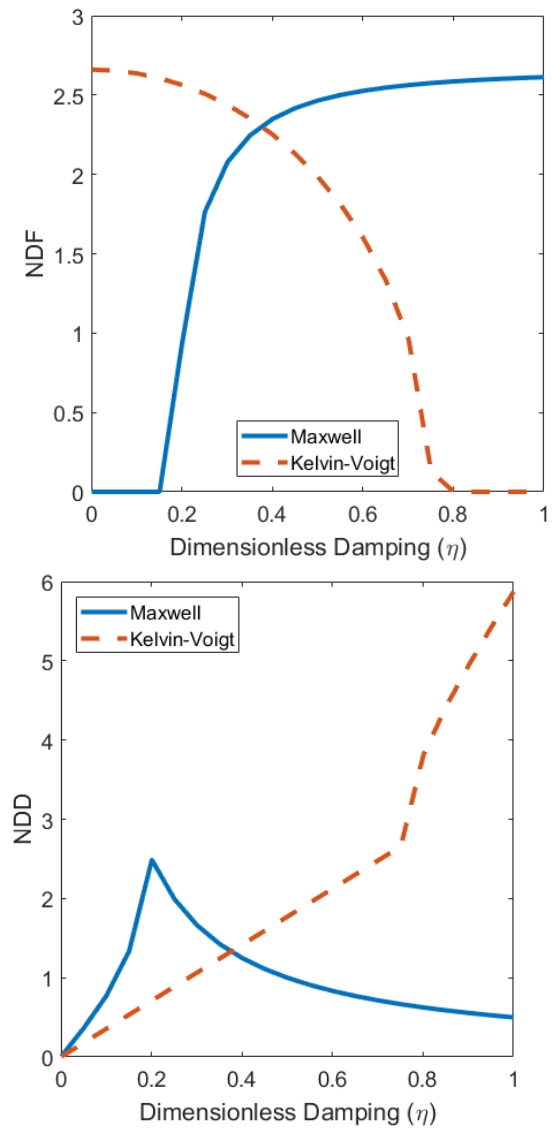

Figure 5. Viscoelastic parameter effect on NDF and NDD of C-C nanotube $\left(\mu=1 \mathrm{~nm}^{2}\right)$

Dimensionles viscoelastic parameter effect on dynamics of viscoelastic nanotube is shown in Figs. (5) and (6). In Kelvin-Voigt material assumption, frequency decreases with 

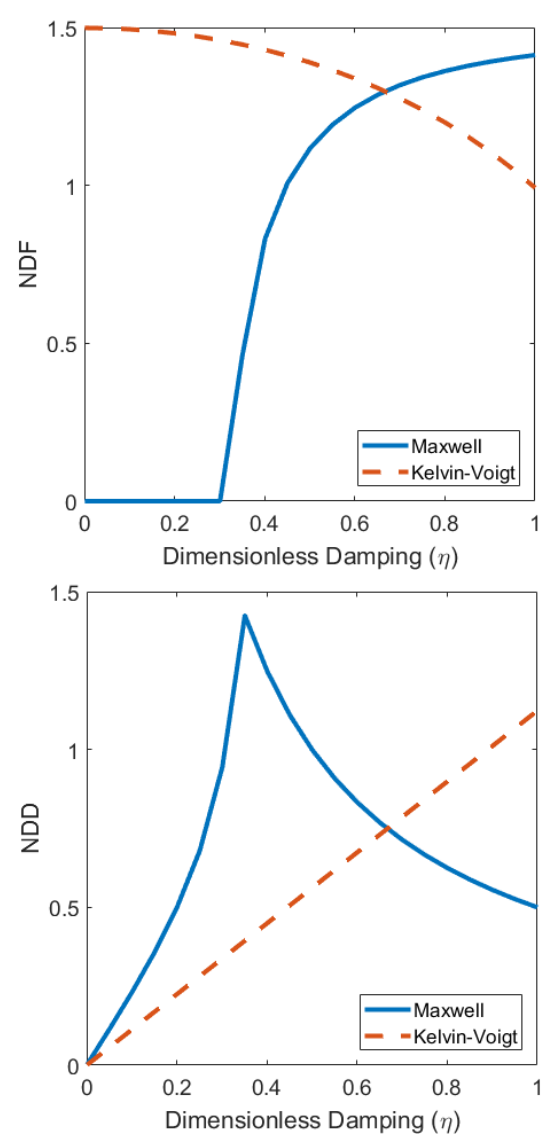

Figure 6. Viscoelastic parameter effect on NDF and NDD of C-F nanotube $\left(\mu=1 \mathrm{~nm}^{2}\right)$
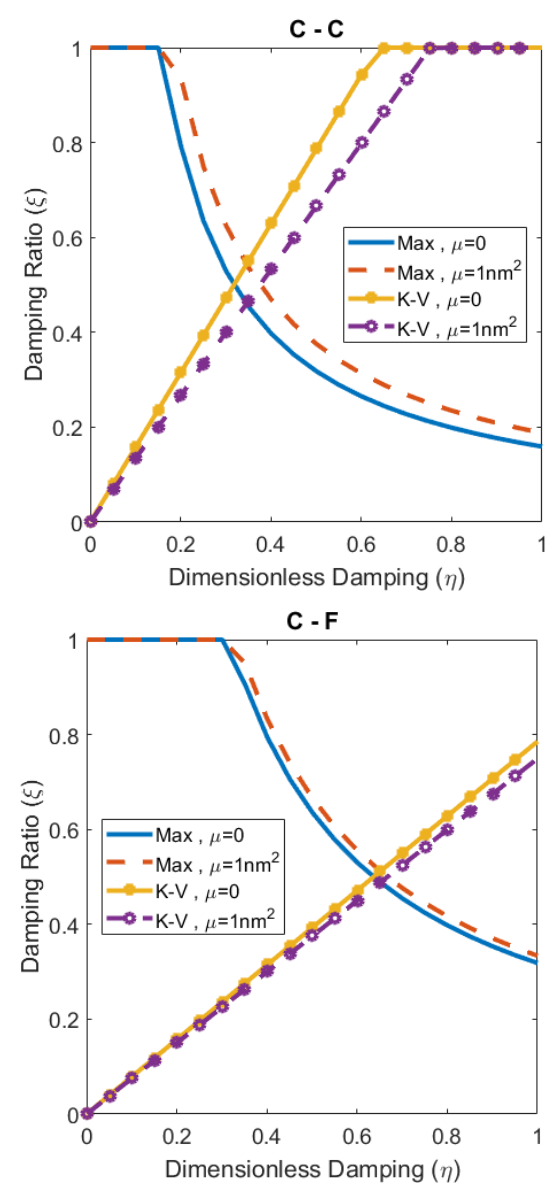

Figure 7. Variation of Damping Ratio with Viscoelastic Parameter enhancing viscoelastic parameter. Damping of Kelvin-Voigt material increases in linear variation characteristics until the frequency drops to zero. In torsional buckling situation, because structural stability loss occurs and no waves propagates through structure, damping increases quadratically. Maxwell material shows different behaviour due to serial connection of viscous damper and spring in modeling. Frequency starts from zero and increases with viscoelastic parameter until the elastic structure modelling frequency.

Damping of nanotube firstly increases when the frequency is zero. Then with increasing viscoelastic parameter, frequency enhances and damping reduces at this time.

In Fig. (7), variation of damping ratio with viscoelastic parameter is depicted. Maxwell material's damping ratio decreases and reversely, Kelvin-Voigt material's damping ratio increases with enhancing viscoelastic parameter. Nonlocal effect enhances the damping ratio in Maxwell material and reduces in Kelvin-Voigt material case.

\section{CONCLUSION}

Present study deals with the torsional dynamic analysis of viscoelastic carbon nanotubes which have been considered as Maxwell and Kelvin-Voigt type viscoelasticity. Governing equation of motion and boundary conditions are obtained with Nonlocal Elasticity Theory. Analytical solution of governing differential equation of motion solved for clamped-clamped and clamped-free boundary cases. Viscoelastic and nonlocal parameters effect on torsional dynamics of viscoelastic CNT are investigated. Results of the present study can be concluded as:

- Maxwell and Kelvin-Voigt type viscoelastic material assumptions have reverse characteristics due to serial or parallel connection modeling.

- Viscoelastic parameter enhances the elasticity of structure and reduces the damping characteristics in Maxwell material.

- Frequency and damping of Kelvin-Voigt material decreases and increases with respect to viscoelasticity parameter.

- Nonlocality shows a softening effect on Kelvin-Voigt material and strengthen effect on Maxwell material.

Present results could be useful for modeling torsional nano devices and products.

\section{REFERENCES}

[1] Eringen, A.C., (1972). Nonlocal polar elastic continua. International Journal of Engineering Science, Doi: 10.1016/0020-7225(72)90070-5.

[2] Eringen, A.C., (1983). On differential equations of nonlocal elasticity and solutions of screw dislocation and surface waves. Journal of Applied Physics 54(9): 4703-10, Doi: 10.1063/1.332803.

[3] Peddieson, J., Buchanan, G.R., McNitt, R.P., (2003). Application of nonlocal continuum models to nanotechnology. International Journal of Engineering Science 41(3-5): 305-12, Doi: 10.1016/ 


\section{S0020-7225(02)00210-0.}

[4] Wang, L., Hu, H., (2005). Flexural wave propagation in single-walled carbon nanotubes. Phys. Rev. B 71(19): 195412, Doi: 10.1103/PhysRevB.71.195412.

[5] Wang, Q., (2005). Wave propagation in carbon nanotubes via nonlocal continuum mechanics. Journal of Applied Physics 98(12): 124301, Doi: 10.1063/1.2141648

[6] Wang, Q., Varadan, V.K., (2006). Wave characteristics of carbon nanotubes. International Journal of Solids and Structures 43(2): 25465, Doi: 10.1016/j.jijsolstr.2005.02.047

[7] Reddy, J.N.N., (2007). Nonlocal theories for bending, buckling and vibration of beams. International Journal of Engineering Science 45(2-8): 288-307, Doi: 10.1016/j.jjengsci.2007.04.004.

[8] Wang, Q., Wang, C.M., (2007). The constitutive relation and small scale parameter of nonlocal continuum mechanics for modelling carbon nanotubes. Nanotechnology 18(7): 075702, Doi: 10.1088/0957-4484/18/7/075702.

[9] Aydogdu, M., (2009). A general nonlocal beam theory: Its application to nanobeam bending, buckling and vibration. Physica E: Low-Dimensional Systems and Nanostructures 41(9): 1651-5, Doi: 10.1016/j.physe.2009.05.014

[10] Ansari, R., Ajori, S., (2015). A molecular dynamics study on the vibration of carbon and boron nitride double-walled hybrid nanotubes. Applied Physics A: Materials Science and Processing 120(4): 1399406, Doi: 10.1007/s00339-015-9324-8.

[11] Numanoğlu, H.M., Akgöz, B., Civalek, Ö., (2018). On dynamic analysis of nanorods. International Journal of Engineering Science 130: 33-50, Doi: 10.1016/j.ijengsci.2018.05.001.

[12] Demir, Ç., Civalek, Ö., (2017). On the analysis of microbeams. International Journal of Engineering Science 121: 14-33, Doi: 10.1016/j. ijengsci.2017.08.016

[13] Avcar, M., Hazim AlSaid Alwan, H., (2017). Free Vibration of Functionally Graded Rayleigh Beam. International Journal Of Engineering \& Applied Sciences 9(2): 127-127, Doi: 10.24107/ijeas.322884.

[14] Chang, W.-J., Lee, H.-L., (2012). Vibration analysis of viscoelastic carbon nanotubes. Micro \& Nano Letters 7(12): 1308-12, Doi: 10.1049/ mnl.2012.0612.

[15] Lei, Y., Adhikari, S., Friswell, M.I., (2013). Vibration of nonlocal Kelvin-Voigt viscoelastic damped Timoshenko beams. International Journal of Engineering Science 66-67: 1-13, Doi: 10.1016/j.jijengsci.2013.02.004

[16] Avcar, M., (2016). Pasternak Zemine Oturan Eksenel Yüke Maruz Homojen Olmayan Kirişin Serbest Titreşimi. Journal of Polytechnic 19(November): 507-12, Doi: 10.2339/2016.19.4.

[17] Karličić, D., Murmu, T., Cajić, M., Kozić, P., Adhikari, S., (2014). Dynamics of multiple viscoelastic carbon nanotube based nanocomposites with axial magnetic field. Journal of Applied Physics 115(23): 234303, Doi: 10.1063/1.4883194

[18] Ghorbanpour Arani, A., Yousefi, M., Amir, S., Dashti, P., Chehreh, A.B., (2015). Dynamic Response of Viscoelastic CNT Conveying Pulsating Fluid Considering Surface Stress and Magnetic Field. Arabian Journal for Science and Engineering 40(6): 1707-26, Doi: 10.1007/ s13369-015-1650-9.

[19] Farokhi, H., Ghayesh, M.H., (2017). Viscoelasticity effects on resonant response of a shear deformable extensible microbeam. Nonlinear Dynamics 87(1): 391-406, Doi: 10.1007/s11071-016-3050-4.

[20] Cajic, M., Karlicic, D., Lazarevic, M., (2015). Nonlocal vibration of a fractional order viscoelastic nanobeam with attached nanoparticle. Theoretical and Applied Mechanics 42(3): 167-90, Doi: 10.2298/ TAM1503167C

[21] Ansari, R., Faraji Oskouie, M., Sadeghi, F., Bazdid-Vahdati, M., (2015). Free vibration of fractional viscoelastic Timoshenko nanobeams using the nonlocal elasticity theory. Physica E: Low-Dimensional Systems and Nanostructures 74: 318-27, Doi: 10.1016/j.physe.2015.07.013.

[22] Zhang, D.P., Lei, Y.J., Wang, C.Y., Shen, Z. Bin., (2017). Vibration analysis of viscoelastic single-walled carbon nanotubes resting on a viscoelastic foundation. Journal of Mechanical Science and Technology 31(1): 87-98, Doi: 10.1007/s12206-016-1007-7.

[23] Zhen, Y., Zhou, L., (2017). Wave propagation in fluid-conveying viscoelastic carbon nanotubes under longitudinal magnetic field with thermal and surface effect via nonlocal strain gradient theory. Modern Physics Letters B 31(8), Doi: 10.1142/S0217984917500695.

[24] Attia, M.A., Mahmoud, F.F., (2017). Analysis of viscoelastic Bernoulli-Euler nanobeams incorporating nonlocal and microstructure effects. International Journal of Mechanics and Materials in Design 13(3): 385-406, Doi: 10.1007/s10999-016-9343-4.

[25] Cajić, M., Karličić, D., Lazarević, M., (2017). Damped vibration of a nonlocal nanobeam resting on viscoelastic foundation: fractional derivative model with two retardation times and fractional parameters. Meccanica 52(1-2): 363-82, Doi: 10.1007/s11012-016$0417-z$.

[26] Wang, J., Shen, H., (2019). Nonlinear vibrations of axially moving simply supported viscoelastic nanobeams based on nonlocal strain gradient theory. Journal of Physics Condensed Matter 31(48), Doi: 10.1088/1361-648X/ab3bf7.

[27] Naghinejad, M., Ovesy, H.R., (2019). Viscoelastic free vibration behavior of nano-scaled beams via finite element nonlocal integral elasticity approach. Journal of Vibration and Control 25(2): 445-59, Doi: $10.1177 / 1077546318783556$

[28] Martin, O., (2019). Nonlocal effects on the dynamic analysis of a viscoelastic nanobeam using a fractional Zener model. Applied Mathematical Modelling 73: 637-50, Doi: 10.1016/j.apm.2019.04.029.

[29] Pavlović, I.R., Pavlović, R., Janevski, G., (2019). Mathematical modeling and stochastic stability analysis of viscoelastic nanobeams using higher-order nonlocal strain gradient theory. Archives of Mechanics 71(2): 137-53, Doi: 10.24423/aom.3139.

[30] Farajpour, A., Ghayesh, M.H., Farokhi, H., (2019). Nonlocal nonlinear mechanics of imperfect carbon nanotubes. International Journal of Engineering Science 142: 201-15, Doi: 10.1016/j.ijengsci.2019.03.003.

[31] Ansari, R., Gholami, R., Ajori, S., (2013). Torsional vibration analysis of carbon nanotubes based on the strain gradient theory and molecular dynamic simulations. Journal of Vibration and Acoustics, Transactions of the ASME 135(5): 1-6, Doi: 10.1115/1.4024208.

[32] Mustapha, K.B., Wong, B.T., (2016). Torsional frequency analyses of microtubules with end attachments. ZAMM Zeitschrift Fur Angewandte Mathematik Und Mechanik 96(7): 824-42, Doi: 10.1002/ zamm.201500007.

[33] Arda, M., Aydogdu, M., (2014). Torsional statics and dynamics of nanotubes embedded in an elastic medium. Composite Structures 114(1): 80-91, Doi: 10.1016/j.compstruct.2014.03.053.

[34] Flügge, W., (1975). Viscoelasticity. Berlin, Heidelberg: Springer Berlin Heidelberg. 